\title{
Spontaneous Bladder Rupture
}

National Cancer Institute

\section{Source}

National Cancer Institute. Spontaneous Bladder Rupture. NCI Thesaurus. Code C115168.

Rupture in the bladder wall due to a pathologic process, in the absence of trauma. 